\title{
MAAT - Sistema de Avaliação de Alunos e Tutores para um Ambiente Virtual de Aprendizagem
}

\author{
Emanuel F. Coutinho ${ }^{1}$, Leonardo O. Moreira $^{1}$, Wellington W. F. Sarmento ${ }^{1}$ \\ ${ }^{1}$ Instituto Universidade Virtual (IUVI) \\ Universidade Federal do Ceará (UFC) \\ Fortaleza - CE - Brasil \\ \{emanuel, leoomoreira, wwagner\}@virtual.ufc.br
}

\begin{abstract}
Learning Management Systems (LMS) are applications that allow interaction between students and tutors, either dynamically or statically for attendance and semi-attendance courses. In the LMS, it is possible to use various learning mechanisms such as visualization of classes, chat tools, message exchange, forums etc. However, the use of these mechanisms does not imply real learning, and this requires the development of an evaluation tool to provide a better understanding of both classes and tutors' efficiency real scenarios. The article presents MAAT system, which allows generation and application of questionnaires in a LMS, and consequent consolidation of results to assist in the evaluation and decision making.
\end{abstract}

Resumo. Ambientes Virtuais de Aprendizagem (AVA) são aplicações que permitem a interação entre alunos e tutores de maneira dinâmica e estática para cursos presenciais e semi-presenciais. No AVA, é possível a utilização de diversos mecanismos de aprendizagem, como visualizar aulas, ferramentas de bate-papo, trocas de mensagens, fóruns etc. Porém, a utilização desses mecanismos não implica o real aprendizado, e para isso é necessário o desenvolvimento de uma avaliação que possa dar maior visibilidade da realidade tanto das aulas quanto da eficiência dos tutores. $O$ artigo apresenta o sistema MAAT, que permite a geração de questionários e aplicação em um AVA, e consequente consolidação dos resultados para auxiliar na avaliação e tomada de decisão.

\section{Introdução}

Os Ambientes Virtuais de Aprendizagem (AVA) integram a tecnologia da informação (TI) e a comunicação, com a finalidade de criar ambientes baseados na Internet que possibilitem o processo de construção de conhecimento e autonomia, por parte de seus interagentes [Castro Filho et al. 2005]. Segundo [Barbosa 2005], os AVA são softwares educativos nos quais os sujeitos podem interagir e construir conhecimento. Conforme [Pequeno et al. 2004], os AVA podem ser divididos quanto ao modelo de interação em:

- Ambientes de apoio a cursos - são orientados ao aluno ou ao professor e voltados para a publicação de textos e atividades direcionadas para cursos à distância;

- Ambientes colaborativos - sua principal característica são trabalhos em grupo e a interação entre os participantes;

- Ambientes híbridos - mesclam características dos anteriores. 
O SOLAR (Sistema Online de Aprendizagem) [Solar 2003] foi desenvolvido pelo Instituto Universidade Virtual, da Universidade Federal do Ceará. É baseado no modelo de três camadas, cujo modelo de participação é orientado ao professor e ao aluno. Quanto ao processamento do sistema, caracteriza-se por ser um sistema distribuído. É orientado ao professor e ao aluno, possibilitando a publicação de cursos e a interação com os mesmos. Além disso, o SOLAR foi desenvolvido potencializando o aprendizado a partir da relação com a própria interface gráfica do ambiente, sendo desenvolvido para que o usuário tenha rapidez no acesso às páginas e ao conteúdo, fácil navegabilidade e compatibilidade com navegadores. Nele, o interagente se sente seguro para explorar os espaços disponibilizados. O ambiente é apoiado numa filosofia de interação e não de controle.

Atualmente, não há uma estratégia de avaliação em AVA que seja eficaz e que possua um retorno mais prático, ou seja, que facilite a tomada de decisão ou realize ações para a melhoria do processo de ensino à distância. Além disso, o feedback às partes envolvidas é demorado, o que torna todo o processo de avaliação defasado e expirado, pois não há tempo hábil para o reajuste do processo de condução das disciplinas nos diversos níveis, nem o simples conhecimento do resultado da avaliação. Além disso, uma avaliação eficaz propicia a redução da evasão nos cursos de graduação devido à adequação de necessidades identificadas, contribuindo para uma melhor ambientação do estudante nos primeiros semestres da graduação e, consequentemente, melhorando a qualidade do ensino e da aprendizagem nos cursos de graduação. Sendo assim, existe a necessidade de avaliação dos tutores, professores e demais perfis que atuam na educação à distância. Esses perfis atuam no acompanhamento das atividades e procuraram evitar problemas, como a evasão de alunos logo que identificada alguma situação, procurando adequar os cursos e perfis de usuários para um melhor rendimento dos alunos nos semestres iniciais da graduação.

A utilização de mecanismos tecnológicos facilitaria todo o processo de avaliação, desde a coleta de informações até análises mais aprofundadas, utilizando técnicas específicas de análise quantitativa e qualitativa. Servindo-se de mecanismos tecnológicos de desenvolvimento de software é possível a construção de um ambiente que possa coletar informações, realizar avaliações, reportar os resultados de forma a permitir uma avaliação mais concisa e precisa. Além disso, outros aspectos que mostram a importância de uma avaliação mais efetiva para a melhoria dos cursos de graduação são:

- Utilização de mecanismos tecnológicos de desenvolvimento de software na construção de um ambiente que possa coletar informações, realizar avaliações, reportar os resultados de forma a permitir uma avaliação mais concisa e precisa;

- Disponibilização de informações de forma mais rápida e detalhada;

- Realização de avaliações mais eficientes;

- Fornecer à tomada de decisão, permitindo ações mais eficazes;

- Dar poio ao planejamento das disciplinas virtuais;

- Identificação de ações para evitar a evasão.

No AVA, é possível o uso de diversos mecanismos de aprendizagem, tais como: visualização de aulas, chats, trocas de mensagens, fóruns etc. Porém, a utilização desses mecanismos não implica no real aprendizado. Para isso, é necessário o desenvolvimento de uma estratégia para avaliação que possa dar maior visibilidade da realidade, tanto das aulas quanto da eficiência dos tutores. Neste sentido, é preciso identificar os fatores a serem avaliados e as dimensões da avaliação que se deseja alcançar. 
O objetivo principal deste trabalho é apresentar o sistema MAAT, que permite a geração de questionários e aplicação no AVA SOLAR, bem como consequente consolidação dos resultados para auxiliar na avaliação e tomada de decisão. Como objetivos secundários, pretende-se: desenvolver a tecnologia utilizada no AVA SOLAR; permitir análises sobre as avaliações e consequentemente a tomada de ações para melhorar o desempenho dos envolvidos e das disciplinas; identificar ações para reduzir a evasão dos alunos; e identificar ações para a adequação do ambiente às necessidades tanto dos alunos quanto dos tutores.

O artigo está dividido, além da introdução, nas seguintes seções: descrição do ambiente, arquitetura da aplicação, sua aplicação e resultados, discussão dos resultados, trabalhos relacionados e conclusão.

\section{Descrição do Ambiente}

Segundo [Wikipedia 2013], na mitologia egípcia, Maet ou Maat é a deusa da justiça e do equilíbrio. O sistema MAAT surgiu da necessidade de se ter uma avaliação sobre os diversos perfis envolvidos no AVA SOLAR. O projeto MAAT é iniciativa de um edital da Universidade Federal do Ceará que subsidia projetos de monitoria de graduação, nos quais os alunos desenvolvem pesquisa aplicada a alguma área de atuação. Desta forma, surgiu a ideia de se desenvolver um módulo de avaliação para o AVA SOLAR.

O acesso ao sistema MAAT se dá por meio de uma URL (Uniform Resource Locator), a partir da tela inicial de acesso, conforme Figura 1, onde o usuário informa sua identificação e senha. Com relação aos usuários, há o cadastro de usuários no menu de cadastros. A tela de abertura do sistema é dividida em duas partes: menu e botões de acesso rápido. No menu, estão itens para a página inicial, os cadastros, os questionários, relatórios e saída da aplicação. Esses mesmos itens estão distribuídos logo abaixo do menu, mas na forma de botões.

Diversos cadastros básicos existem no sistema: usuário, categoria, subcategoria, questão, resposta, e um cadastro mais complexo, que é o de questionário. A tela inicial de cada cadastro é uma tela intermediária de acesso. Nela é possível visualizar os registros conforme alguns filtros pré-definidos para cada cadastro, inserir um novo registro, alterar e excluir um registro selecionado. Também, na tela inicial, é possível a aplicação dos questionários a turmas e a visualização dos resultados sob o formato de relatórios. Além disso, alguns relatórios podem ser gerados em formatos diferentes, que é uma necessidade dos funcionários, e que facilita a utilização dos dados do formulário em ferramentas de cálculo estatístico ou de banco de dados.

\section{Arquitetura e Aspectos de Implementação}

O sistema foi desenvolvido para prover o acesso de algumas pessoas que trabalhassem com avaliação ou necessitassem que algum questionário específico fosse aplicado aos alunos dos cursos semi-presenciais. Assim, o sistema deveria se comunicar com o AVA SOLAR por meio de algum mecanismo de integração. O MAAT foi projetado para ser um software modularizado e independente dos AVA que irão se servir da sua utilização. Com isso, o projeto de independência facilita a integração do MAAT com algum outro AVA. Neste sentido, um conjunto de serviços Web [Pautasso et al. 2008] foi implementado para 


\section{Apuda Conkato \\ sistema de avaliação}

Home

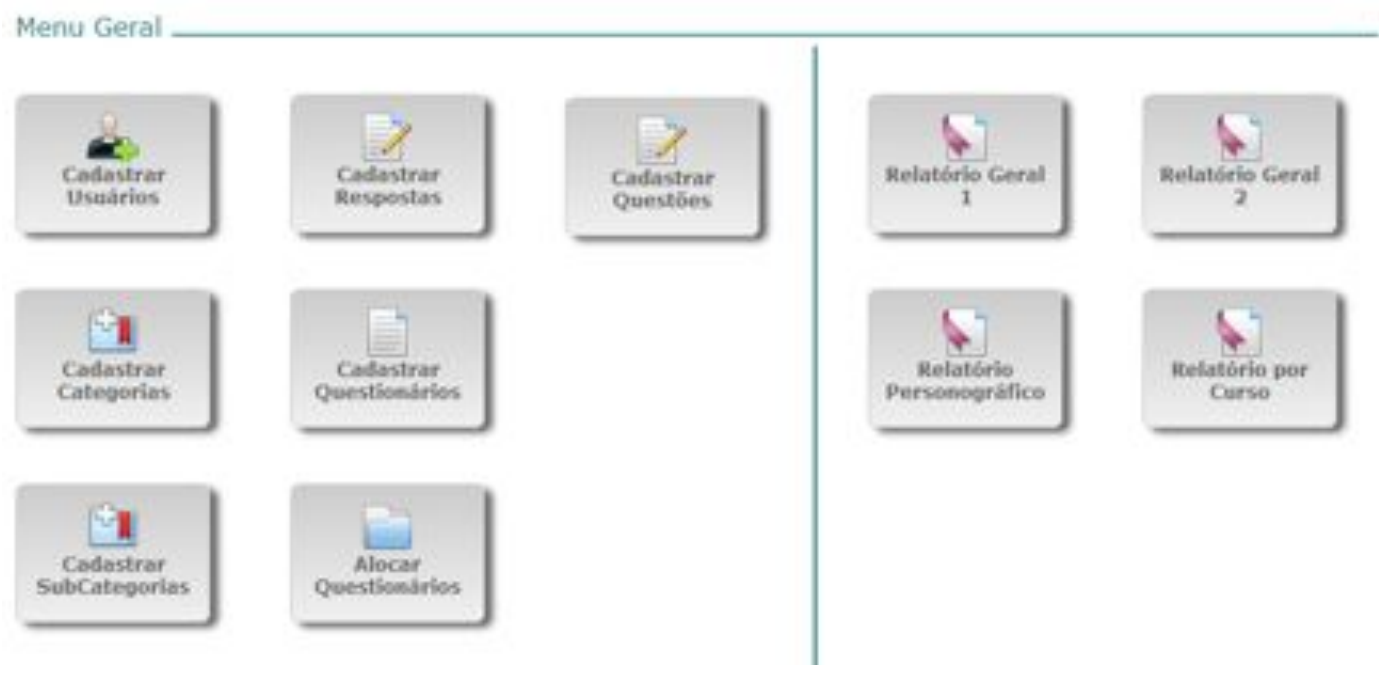

Figura 1. Menu inicial do MAAT com funcionalidades de cadastros, elaboração de questionários e relatórios

que o MAAT e o AVA possam trocar informações sem gerar dependências. A Figura 2 ilustra os componentes arquiteturais do MAAT e suas interações com o AVA.

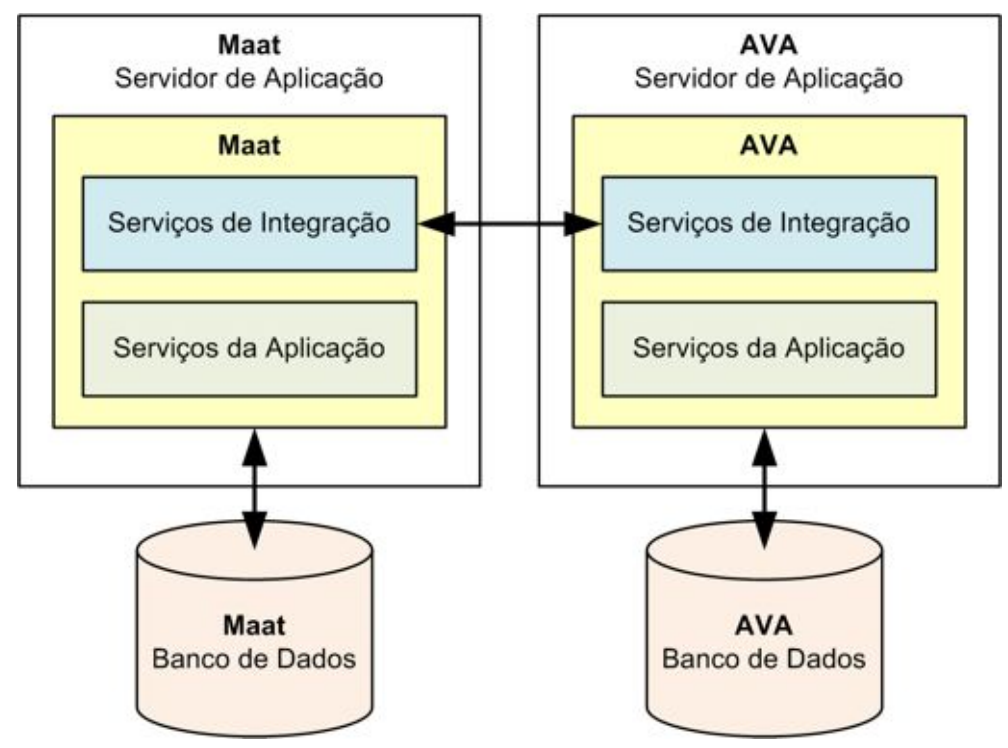

Figura 2. Componentes arquiteturais do MAAT e suas interações

A fim de prover portabilidade ao MAAT optou-se por utilizar a plataforma Java [Oracle 2013b] para seu desenvolvimento. A tecnologia de serviços Web 
[Pautasso et al. 2008] pode ser utilizada para prover integração de softwares por meio do modelo requisição/resposta. Esta tecnologia agrega uma série de vantagens, tais como a utilização de padrões de projeto, facilidade de implementação e manutenção. Além disso, disponibiliza o recurso para integração de novos serviços, fornecendo a extensibilidade do software.

A arquitetura do MAAT exige processamento tanto do lado do cliente quanto do servidor. Isso é vantajoso por eliminar processamento desnecessário do lado do servidor, fornecendo assim melhor desempenho da ferramenta. Para isso, críticas de entradas de dados, formatação e organização das respostas são feitas do lado do cliente por meio da linguagem JavaScript [W3CSchools 2013b]. Todas as requisições que partem dos clientes são realizadas por meio de requisições AJAX [W3CSchools 2013a]. A utilização de recursos AJAX fornece interfaces visuais mais amigáveis, rápidas e poderosas, além de eliminar carregamentos desnecessários de partes ou códigos das páginas Web.

O fato do MAAT ser executado e gerenciado por um Java Web Container, surgem algumas vantagens como, por exemplo, a responsabilidade pelo ciclo de vida dos componentes Web, otimizações, uma série de serviços adicionais, e facilidades de instalação e atualização dos aplicativos. Um Java Web Container é um software que possibilita que um aplicativo Java para Web possa ser disponibilizado na Web. O projeto MAAT foi desenvolvido pensando na independência. Portanto, o MAAT pode ser gerenciado por qualquer Java Web Container que tenha suporte a serviços Web como, por exemplo, o Glassfish [Oracle 2013a].

Para ser utilizado no AVA SOLAR, o MAAT teve que ser integrado e seu conjunto de serviços Web foi implementado para possibilitar a troca de informações com o AVA. Para que os questionários aparecessem no SOLAR, após a identificação do usuário, foram necessários a inclusão de alguns critérios que invocavam os serviços Web do MAAT para a geração da página de questionário. Neste sentido, todo AVA que quiser utilizar o MAAT, deve fazer essa mesma alteração. Com isso, o SOLAR pode invocar o MAAT como se fosse um recurso próprio. A única função invocada pelo AVA é aquela que chama a tela do questionário quando um usuário se identifica no AVA. Vale ressaltar que essa tela só aparece caso o usuário deva preencher algum questionário. Ao ser respondido, a aplicação salva o resultado na base de dados do MAAT para que esse possa gerar os relatórios, conforme representado pela seta entre o MAAT e o AVA, na Figura 2.

\section{Aplicação da Ferramenta e Resultados}

Após o desenvolvimento do sistema, seguido de testes e validação com equipe de avaliação envolvida no projeto, foram modelados e implementados os primeiros questionários. Foram construídos dois questionários para aplicação às turmas dos cursos semi-presenciais da UAB (Universidade Aberta do Brasil), que correspondem aproximadamente a 3700 alunos matriculados e que estão cursando alguma disciplina. A aplicação possui a capacidade de criar questionários a qualquer momento; porém, nem todos os relatórios disponíveis são genéricos.

O primeiro questionário elaborado, nomeado de "Distribuição de Disciplinas", foi aplicado às turmas de todos os cursos. O segundo, denominado de "Personográfico", foi aplicado às turmas de Educação à Distância, disciplina do primeiro semestre e a primeira com que os alunos tinham contato, que contemplava cerca de 1500 alunos. 


\subsection{Relatório Geral}

Este relatório permite a visualização de informações relativas a questionários aplicados. É um relatório quantitativo onde é possível visualizar informações sobre a quantidade de cursos, disciplinas, polos, tutores, alunos, categorias, sub-categorias e períodos. O relatório propõe uma visão macro de todas as dimensões da avaliação. A ideia que permeia é gerar informações em alto nível para facilitar a interpretação dos outros relatórios que são mais minuciosos e detalhistas. O Relatório Geral possui visões para os dois questionários aplicados e retorna aos mesmos campos, porém com resultados diferentes. Geralmente sua utilização é em conjunto com outros relatórios, como auxílio de uma visão mais genérica para algum aspecto específico de um relatório em particular. A Figura 3 exibe este relatório nos formatos HTML, PDF e XLS, que são gerados separadamente conforme a necessidade. A diferenciação de formatos é uma necessidade dos vários funcionários que utilizam os dados para alguma outra funcionalidade.

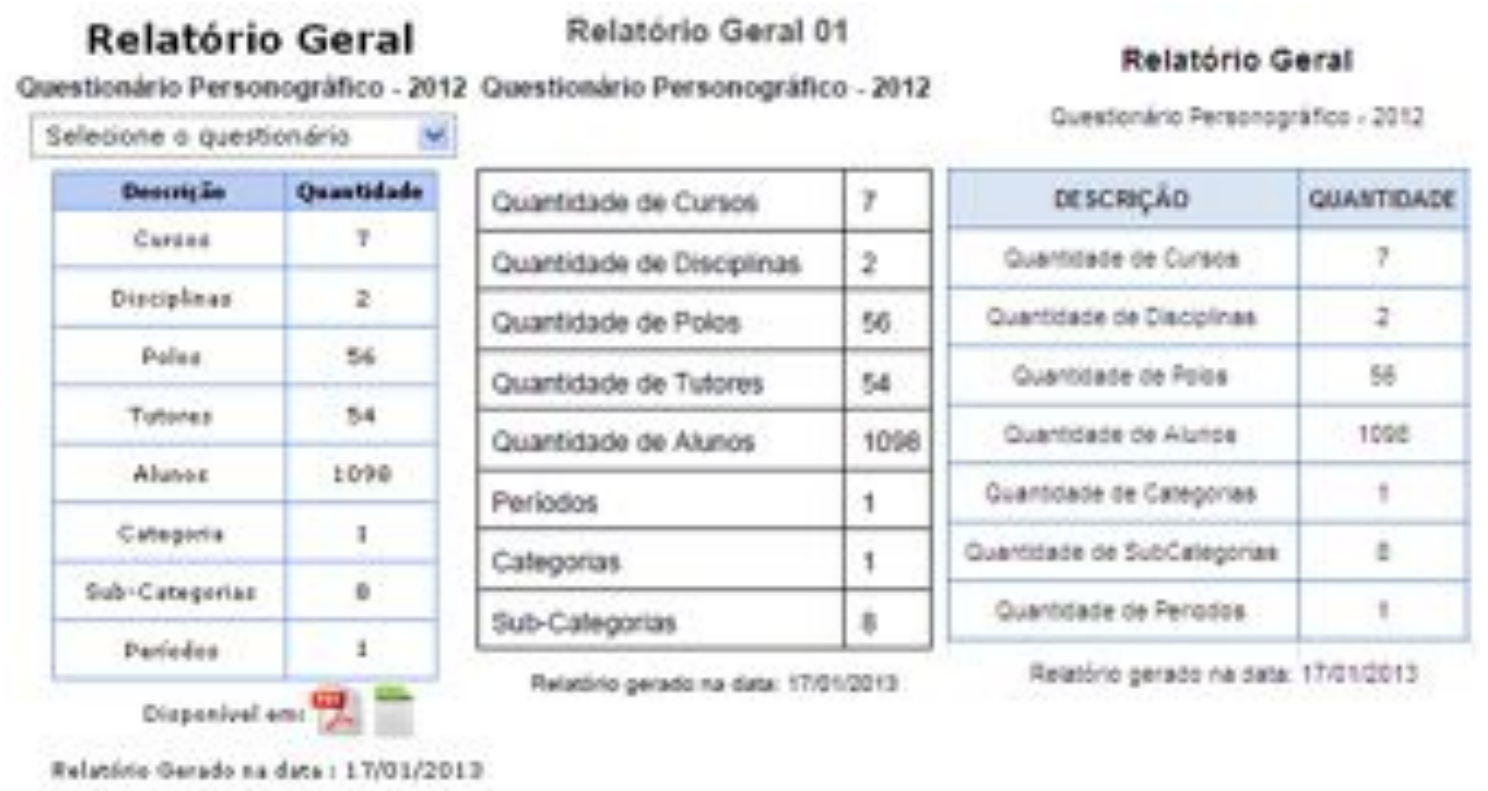

Figura 3. Relatório geral

\subsection{Relatório Geral por Curso}

Esse relatório se refere aos dois questionários aplicados. Nele é possível visualizar, para cada curso e por cada uma das disciplinas, suas turmas (polos), e os respectivos tutores com a quantidade de alunos, além de totalizadores. A diferença entre os dois questionários é que o personográfico foi aplicado apenas às turmas de Introdução à Educação à Distância, referindo-se apenas a uma disciplina, enquanto que no questionário de distribuição de disciplinas é possível se visualizar todas as disciplinas do curso. Por meio desse relatório é possível identificar quais cursos estão com muita, ou pouca procura, quais cursos estão sem tutores e quais tutores estão sobrecarregados. A Figura 4 exibe este relatório.

\subsection{Relatório Personográfico}

Disponível apenas no formato XLS (formato de planilha eletrônica do Microsoft Excel), este relatório foi gerado apenas para o questionário personográfico. É selecionado o curso 


\section{Relatório Geral 2}

Questionário Personográfico - 2012

Selecione o questionario

Selecione o curso

\begin{tabular}{|c|c|c|c|c|c|c|}
\hline Cerso & \multicolumn{6}{|c|}{ AAAAA } \\
\hline \multicolumn{7}{|c|}{ I Sisciplina(s) XX00001 - EBUCACAO A DISTANCIA } \\
\hline Discipline & & & & & & Total \\
\hline Turmas & POLO 1 & POLO2 2 & pono 3 & POCOA & E000 5 & 5 \\
\hline Tutar & 9 & FU ANO DE TML 1 & FUANO DE TML 2 & ALANO DE IAL 3 & FULANO DE TAL 4 & 5 \\
\hline Alunós & 12 & 21 & 2 & 18 & 11 & 54 \\
\hline Periads & 1 & 1 & 1 & 1 & 1 & 5 \\
\hline
\end{tabular}

Figura 4. Relatório geral por curso

desejado e é gerada uma planilha, contendo a consolidação em valores absolutos e percentuais de cada questão por curso. Mediante este questionário é possível se traçar um perfil sócio-econômico dos cursos. Informações de sexo, faixa etária, estado civil, grau de instrução, horas de trabalho e estudo, entre outras, podem ser obtidas a partir do relatório. A Figura 5 exibe este relatório. O questionário é utilizado por diversas equipes como entrada para outros processos da instituição, análise de informações sócio-econômicas e desempenho dos alunos.

\begin{tabular}{|c|c|c|c|c|}
\hline QT & $\begin{array}{l}\text { Qual é o nùmero de horas que vocé traballha per } \\
\text { semana? }\end{array}$ & & & \\
\hline & Itso trabaho & $\begin{array}{l}\text { Marios de } 20 \\
\text { horas por zamans }\end{array}$ & $\begin{array}{l}\text { De } 20 \text { * } 30 \text { herrat } \\
\text { semanas }\end{array}$ & $\begin{array}{l}\text { Acims de } 30 \\
\text { horas }\end{array}$ \\
\hline Totais: & 33 & $16-2$ & 25 & 102 \\
\hline Porcentagens & it.7s & 909 & 1420 & 57.56 \\
\hline \multirow[t]{2}{*}{ Q8 } & $\begin{array}{l}\text { Indique as formas de acesso ao ambiente virtual de } \\
\text { aprendizagem. }\end{array}$ & & & \\
\hline & Trabalho & Cass & Polo & Ostros \\
\hline Totais: & 25 & 114 & 8 & 29 \\
\hline Porcentapens: & 1420 & 64.77 & 465 & 16.48 \\
\hline \multirow[t]{2}{*}{$Q 9$} & $\begin{array}{l}\text { Gual é o tempo que vocé dedica por dia para o } \\
\text { estudo, fora do ambieme virtual de aprendizagem? }\end{array}$ & & & \\
\hline & Wanhuma & Manas de 1 hara & Emre 1 e 2 houss & $\begin{array}{l}\text { Entele } 2+3 \\
\text { nors }\end{array}$ \\
\hline Totak: a & 2. & 30 & 101 & 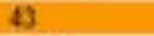 \\
\hline Porcentagens: & 1.14 & 17.05 & 57.39 & 24.43 \\
\hline \multirow[t]{2}{*}{ Q10 } & $\begin{array}{l}\text { Quantas horas por dia vocé dedica as essuds a } \\
\text { dintancis conectado(s) mum ambiente virtasl de } \\
\text { aprendizagem? }\end{array}$ & & & \\
\hline & Itenhuma & Manos de 1 hora & Emie 1 e 2 horas & $\begin{array}{l}\text { Ente } 2+3 \\
\text { horns }\end{array}$ \\
\hline Totaix: & 1 & 22 & 102 & $51 \quad$ \\
\hline Porcentagens & 057 & 1250 & 5796 & 20.90 \\
\hline
\end{tabular}

Figura 5. Relatório personográfico

\subsection{Quadro Resumo de Respostas}

Aplicado ao questionário de distribuição de disciplinas, e disponível apenas em XLS. Para a utilização deste relatório, o avaliador escolhe um curso, e as respostas dos alunos 
de todas as turmas do curso selecionado são consolidadas por semestre, permitindo uma visão geral do curso. A Figura 6 exibe um trecho do relatório contendo apenas quatro semestres.

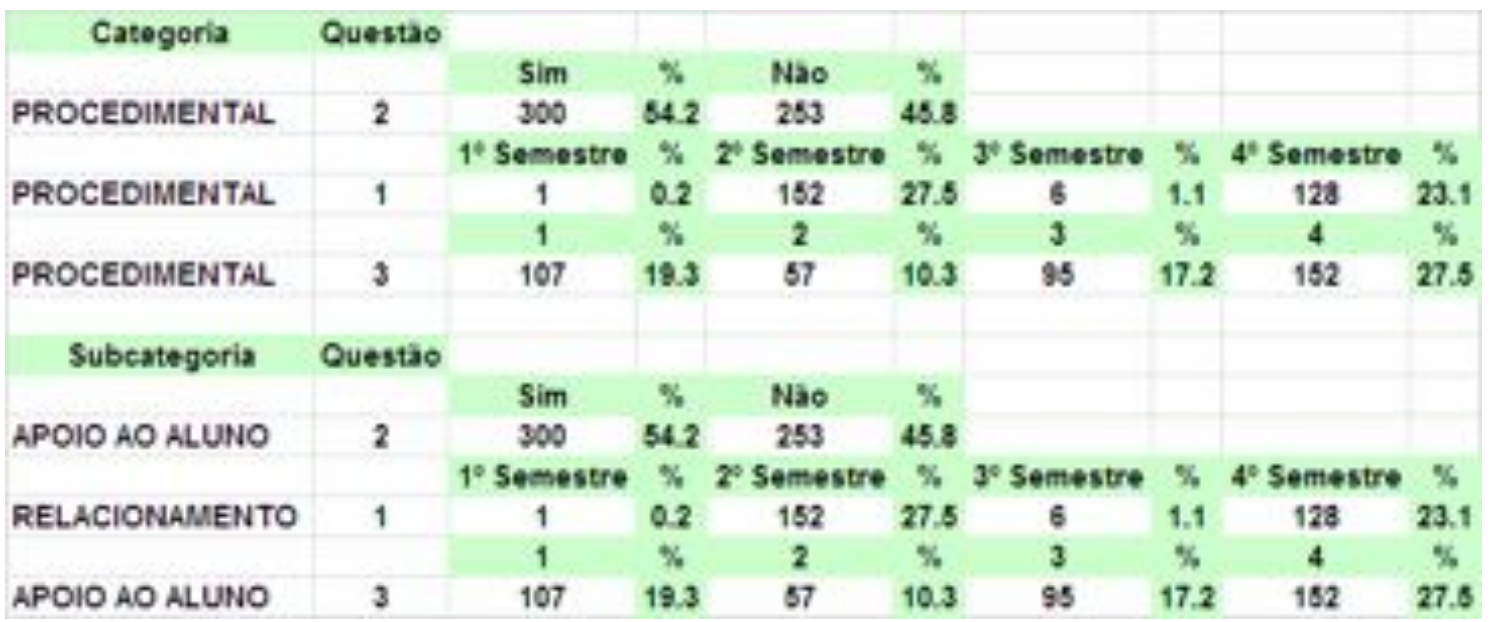

Figura 6. Quadro resumo de respostas

\subsection{Discussão dos Resultados}

Uma vez que relatórios são gerados, o conjunto de informações tabuladas estão prontas para serem utilizadas. O momento é da análise dos dados para o entendimento da situação de aplicação dos questionários e, consequentemente, tomada de decisão. Alguns relatórios são complementares e mostram visões diferentes do mesmo aspecto. Ao cruzar as informações obtidas nos relatórios é possível identificar deficiências nos polos tanto do ponto de vista de suporte aos cursos quanto aos alunos. Por exemplo, o desempenho dos alunos em relação ao desempenho dos tutores.

O relatório geral exibe informações quantitativas totalizadoras de cursos, alunos e tutores, sem distinção. O relatório geral por curso exibe as informações dos tutores por curso e a quantidade de alunos. Assim, é possível identificar a relação entre o quantitativo de alunos por tutor e a quantidade total de alunos. Consequentemente, distorções entre as proporções de alunos por tutor podem ser identificadas.

Analisando o relatório personográfico, é possível avaliar as turmas iniciais do curso, pois todos os alunos cursam a disciplina de Educação à Distância. Desta forma, é possível atingir todos os alunos que iniciaram os cursos. Sendo aplicado ao longo do curso é fácil identificar a evolução da dedicação do aluno ao curso pelas horas dedicadas ao estudo, por exemplo. Assim, é possível avaliar a necessidade de um maior apoio aos alunos em horários diferentes do horário das aulas.

Apesar das informações estarem nos relatórios, a avaliação ainda é subjetiva. Os relatórios apenas apóiam a tomada de decisão baseada em suposições. Um mecanismo de avaliação mais detalhado é necessário para melhor avaliação dos alunos, tutores e disciplinas. Subsidiar o planejamento de atividades é uma das vantagens da aplicação. Por exemplo, o planejamento de novas turmas em polos diferentes baseados em dados numéricos como quantidade de alunos cursando e a demanda. Os relatórios gerais e por curso apóiam esse tipo de planejamento. A reformulação das turmas, podem gerar a união 
de turmas com poucos alunos, ou mesmo a extinção; porém, em localidades próximas, evitar gastos com turmas pequenas também pode ser uma consequencia dessa análise.

Um fato positivo da aplicação é a capacidade de reaplicação de um questionário. Não é necessário reconstruir o questionário já que a ferramenta favorece a reutilização e reaplicação de questionários existentes. Isso permite que se identifique a evolução de algum aspecto retratado no questionário e se realize alguma ação. Apesar dessa capacidade de geração e aproveitamento de questionários a qualquer momento, a aplicação não possui a geração dos relatórios também para todos os questionários. Atualmente alguns relatórios são específicos para determinado relatório, por questões de informação e formatação da tela.

O sistema MAAT ainda não realiza avaliações dos professores, normalmente realizadas ao final do semestre letivo; ele apenas disponibiliza informações. Uma atividade que auxiliaria muito seria o tratamento estatístico dos dados, podendo revelar ainda mais padrões ou novas ações. A ideia é que questionários sejam elaborados para prover essa avaliação para os alunos dentro do AVA, e sua consolidação mais ágil. Assim, os resultados serão mais rápidos de serem analisados e os professores podem ter acesso as informações e replanejar suas futuras turmas.

\section{Trabalhos Relacionados}

Diversas formas de avaliação poderm ser utilizadas mediante mecanismos computacionais. Em [Demarco et al. 2012], foi utilizada uma ferramenta web de survey, o LimeSurvey, para a realização da avaliação dos tutores de um curso de especialização. As respostas foram analisadas por meio de estatísticas simples (percentuais e médias) com o auxílio do pacote estatístico SPSS. No caso do MAAT, a análise ainda é manual, servindo-se dos resultados.

Diversos trabalhos tratam da avaliação de AVAs em determinado aspecto. Alguns trabalhos tratam de metodologias para avaliação em AVAs. Um exemplo é o trabalho de [Chagas et al. 2011], onde é apresentada uma metodologia para avaliação de AVAs a ser usada por instituições, professores ou desenvolvedores que necessitam verificar o impacto da usabilidade, comunicabilidade e sociabilidade em seus cursos. No trabalho de [Tijiboy et al. 2009] são abordadas formas de mediação na ferramenta de fórum de discussão pela definição de categorias de mediação, focalização, expansão, significação, afetividade, recompensa, regulação, gerenciamento e reflexão.

[Roque et al. 2011] apresentam os resultados de uma pesquisa com professores e alunos em um curso à distância com o objetivo de provocar reflexões sobre a qualidade dos cursos nessa modalidade, servindo-se de uma análise qualitativa com o auxílio da ferramenta ALCESTE. [Mateus et al. 2011] desenvolvem um módulo adaptativo para um AVA, onde conforme o desempenho do aluno ele se ajusta, alterando desafios pedagógicos. Assim, é possível analisar o andamento do estudante no processo de aprendizagem.

Em [Oliveira et al. 2009], é realizada uma avaliação somativa em que um sistema online de perguntas e respostas retorna uma resposta adequada para o aluno, podendo este chegar a respostas corretas, desde que saiba realizar as perguntas. [Caldas and Favero 2009] propuseram uma ferramenta que, por meio de técnicas de inteligência artificial, realiza uma avaliação tanto quantitativa, objetivando o auxílio ao 
docente quanto qualitativa, visando o aprendizado do estudante sobre mapas conceituais. [Dillenburg and Teixeira 2011] apresentam a proposta para um modelo qualitativo de avaliação de AVAs baseados em conceitos de aprendizagem, tecnologia e software. O foco é no ambiente. [Rocha et al. 2011] apresentam o SISAVI, uma ferramenta para automatizar o processo avaliativo por parte dos diversos atores que participam do processo educacional: alunos, professores e equipe multidisciplinar.

No trabalho de [Manhães et al. 2012] técnicas de mineração de dados são utilizadas para para identificar problemas relacionados a alunos que não conseguem completar seus cursos de graduação. Em relação ao MAAT, a ideia é a mesma: analisar cursos de graduação, porém com técnicas diferentes. A mineração de dados é uma ideia a ser considerada por causa dos seus resultados. [Amorim et al. 2008] utilizam técnicas de aprendizado de máquina aplicadas na previsão da evasão, para entender os motivos de evasão universitária. Os dados foram obtidos de uma instituição de ensino superior, e não de um AVA, e aplicados na ferramenta WEKA para classificação. Em [Penedo and Capra 2012], um processo de descoberta de conhecimento útil é descrito para um sistema de EAD com intuito de investigar as informações relevantes que auxiliem na identificação do padrão dos usuários que utilizam o sistema. O MAAT também tem essa intenção, ou seja, identificar padrões por meio de questionários e, consequentemente, de relatórios para que possam ser tomadas ações.

\section{Conclusão}

A realidade dos cursos semi-presenciais é diferente daqueles presenciais. A infraestrutura e a dinâmica de cada curso são variadas; portanto, os mecanismos de avaliação devem também ser diferentes. O sistema MAAT tem a intenção de subsidiar a avaliação dos cursos semi-presenciais, mas também é possível ser utilizado em cursos presenciais. $\mathrm{O}$ módulo de geração de questionários permitiu a criação e aplicação de avaliações entre alunos e tutores, e consequentemente uma consolidação mais ágil.

Sua aplicação no AVA SOLAR foi de extrema utilidade, mesmo que ainda em um estágio inicial e aplicado há pouco tempo (apenas um semestre), pois diversos aspectos positivos e negativos foram identificados, permitindo uma tomada de decisão para a melhoria das turmas e dinâmica dos cursos semi-presenciais. Outros benefícios foram: maior agilidade na consolidação dos resultados dos questionários, flexibilidade para pesquisas e criação, e aplicação de novas avaliações.

Uma dificuldade inicial foi a comunicação entre equipe de desenvolvimento e equipe especializada em avaliação. Muitas vezes os termos utilizados não eram os mesmos, sendo necessário um nivelamento. Mudanças constantes nos requisitos, mesmo sem estes serem desenvolvidos ou estáveis, muitas vezes atrasaram o projeto. Entende-se que é natural a mudança nos requisitos, mas deve haver um controle de mudanças mais formal para evitar muito trabalho desnecessário. Neste aspecto, uma prática melhor definida para especificação dos requisitos e controle de alterações teria auxiliado no projeto, evitando repetição de trabalho e problemas de comunicação. A integração entre os dois sistemas (MAAT e SOLAR) necessitou certo esforço, pois são dois ambientes com plataformas distintas.

Como trabalhos futuros pretende-se a aplicação dos questionários para avaliação de alunos e tutores de maneira mais dinâmica e sistematizada em cursos semi-presenciais 
e presenciais, bem como a criação de um mecanismo de avaliação quantitativo, em que uma nota seria dada baseada nos resultados. Para isso, questionários deverão ser bem planejados para permitir a visibilidade de vários aspectos de avaliação docente, como assiduidade, habilidade nas aulas, exercícios etc. A avaliação das atividades dos professores pelos alunos é o próximo passo a ser realizado, de maneira que estas se tornem mais ágeis e aplicadas sistematicamente, subsidiando a tomada de decisão e avaliação mais precisa. O MAAT foi integrado ao AVA SOLAR. Pretende-se, também, integrar com outros AVA de código livre, tais como o Moodle [Moodle 2013] e o iVeLA [Moreira et al. 2009]. E assim, verificar o impacto de integração com outros AVA.

\section{Agradecimentos}

Agradecemos à Pró-Reitoria de Graduação (PROGRAD) pelo financiamento do projeto "Desenvolvimento do Sistema de Avaliação do Ambiente Virtual de Aprendizado SOLAR". Também agradecemos aos funcionários, bolsistas e estagiários do Centro de Produção II que apoiaram o desenvolvimento do sistema MAAT. Agradecemos ao Setor de Avaliação (SAV) pelo suporte relacionado à avaliação de cursos. Finalmente, somos gratos ao Instituto Universidade Virtual (IUVI) pelo incentivo ao desenvolvimento de novas soluções.

\section{Referências}

Amorim, M. J. V., Barone, D., and Mansur, A. U. (2008). Técnicas de aprendizado de máquina aplicadas na previsão de evasão acadêmica. In XIX Simpósio Brasileiro de Informática na Educação (SBIE 2008).

Barbosa, R. M. (2005). Ambientes Virtuais de Aprendizagem. Editora Artmed.

Caldas, V. M. and Favero, E. L. (2009). Uma ferramenta de avaliação automática para mapas conceituais como auxílio ao ensino em ambientes de educação a distância. In XX Simpósio Brasileiro de Informática na Educação (SBIE 2009).

Castro Filho, J. A., Loureiro, R. C., Paula, P. S., Sarmento, W. W. F., Peixoto, L. E., Pequeno, H. S. L., Rocha, B. T. S., and Viana Júnior., G. S. (2005). Portal humanas: Um ambiente colaborativo para criação de projetos e comunidades virtuais para a área de humanidades. In XVI Simpósio Brasileiro de Informática na Educação (SBIE 2005).

Chagas, D. A., Lisboa, R. P., and Furtado, E. S. (2011). Framework maava - metodologia de avaliação de ambientes virtuais de aprendizagem. In XXII Simpósio Brasileiro de Informática na Educação (SBIE 2011).

Demarco, D. J., Antunes, E. D. D., Pinheiro, I. A., and Brunetta, N. (2012). Avaliação da tutoria no curso de especialização em negociação coletiva / modalidade a distância (mpog-ea/ufrgs). RENOTE - Revista Novas Tecnologias na Educação, 10(3).

Dillenburg, D. J. and Teixeira, A. C. (2011). Uma proposta de avaliação qualitativa em ambientes virtuais de aprendizagem. In XXII Simpósio Brasileiro de Informática na Educação (SBIE 2011).

Manhães, M., da Cruz, S. M. S., Costa, R., Zavaleta, J., and Zimbrao, G. (2012). Identificação dos fatores que influenciam a evasão em cursos de graduação através de sistemas baseados em mineração de dados: Uma abordagem quantitativa. In VIII Simpósio Brasileiro de Sistemas de Informação (SBSI 2012). 
Mateus, G. P., Wilges, B., Nassar, S. M., Wronscki, V., and Bastos, R. C. (2011). Um módulo adaptativo para ava baseado no desempenho do estudante. In XXII Simpósio Brasileiro de Informática na Educação (SBIE 2011).

Moodle (2013). Moodle. https://moodle.org/. Online; acessado em janeiro-2013.

Moreira, L. O., Silva, J. C., Damico, J. R. O., Morais, M. A. H. L., Almeida, R. F., and Machado, J. C. (2009). ivela - internet voice e-learning application. In 9th IFIP World Conference on Computers in Education (WCCE 2009).

Oliveira, M. G., Oliveira, E., and Marchesi, R. Z. (2009). Um qasystem para interação de alunos em avaliações somativas a distância. In XX Simpósio Brasileiro de Informática na Educação (SBIE 2009).

Oracle (2013a). Glassfish application server. http://glassfish.java.net/. Online; acessado em janeiro-2013.

Oracle (2013b). Java ee at a glance. http://www.oracle.com/technetwork/java/javaee/index.html. Online; acessado em janeiro-2013.

Pautasso, C., Zimmermann, O., and Leymann, F. (2008). Restful web services vs. "big"” web services: making the right architectural decision. In Proceedings of the 17th international conference on World Wide Web, WWW'08, pages 805-814, New York, NY, USA. ACM.

Penedo, J. and Capra, E. (2012). Mineração de dados na descoberta do padrão de usuários de um sistema de educação à distância. In VIII Simpósio Brasileiro de Sistemas de Informação (SBSI 2012).

Pequeno, M. C., Silva, C. O., and Loureiro, R. C. (2004). Modelo para gestão e implementação de ambientes virtuais de aprendizagem numa perspectiva de interface adaptativa. In VIII Congreso de Educación a Distancia (CREAD MERCOSUL 2004).

Rocha, E. M., Joye, C. R., Joye, F. M., and Werlang, R. (2011). A automação da avaliação institucional na ead. In XXII Simpósio Brasileiro de Informática na Educação (SBIE 2011).

Roque, G. O. B., Campos, G. H. B., Ziviani, C., and Fonseca, M. V. A. (2011). Qualidade em ead: o que pensam os cursistas? In XVII Workshop de Informática na Educação (WIE 2011).

Solar (2003). Solar. http://www.solar.virtual.ufc.br/. Online; acessado em janeiro-2013.

Tijiboy, A. V., Carneiro, M. L. F., Woiciechoski, L. R., and Pereira, E. A. (2009). Compreendendo a mediação do tutor a distância. RENOTE - Revista Novas Tecnologias na Educação, 7(1).

W3CSchools (2013a). Ajax tutorial. http://www.w3schools.com/ajax/default.asp. Online; acessado em janeiro-2013.

W3CSchools (2013b). Javascript tutorial. http://www.w3schools.com/js/default.asp/. Online; acessado em janeiro-2013.

Wikipedia (2013). Maet. http://pt.wikipedia.org/wiki/Maet/. Online; acessado em janeiro2013. 